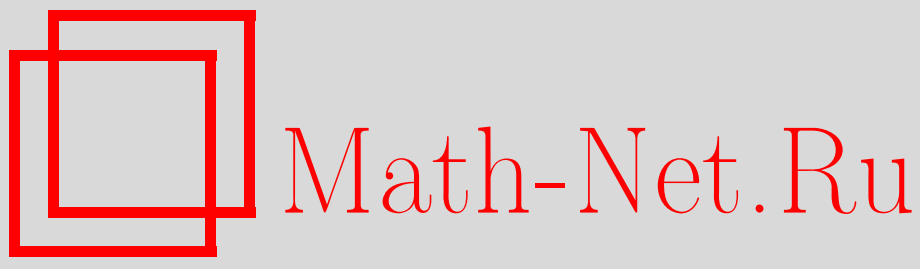

В. П. Маслов, А. С. Черный, О минимизации и максимизации энтропии в различных дисциплинах, Теория вероятн. и ее примен., 2003, том 48, выпуск 3, 466-486

DOI: https://doi.org/10.4213/tvp266

Использование Общероссийского математического портала MathNet.Ru подразумевает, что вы прочитали и согласны с пользовательским соглашением

http://www . mathnet.ru/rus/agreement

Параметры загрузки:

IP: 44.207 .124 .84

26 апреля 2023 г., 11:09:13

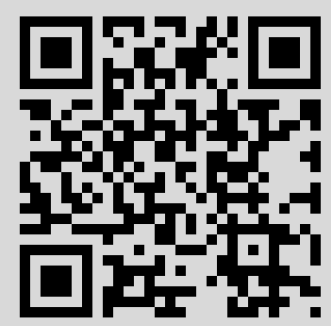


(C) 2003 г.

\author{
МАСЛОВ В. П., ЧЕРНЫЙ А. С.**
}

\title{
О МИНИМИЗАЦИИ И МАКСИМИЗАЦИИ ЭНТРОПИИ В РАЗЛИЧНЫХ ДИСЦИПЛИНАХ
}

В работе рассматривается ряд проблем, связанных с минимизацией относительной энтропии при линейных ограничениях. Обсуждается связь этой задачи со статистической физикой, теорией информации и финансовой математикой. В разделе, относящемся к финансовой математике, мы приводим явный вид ближайшей по энтропии мартингальной меры в общей многошаговой модели эволюции цен. Помимо этого, дается явное решение задачи максимизации экспоненциальной полезности в общей многошаговой модели эволюции цен.

Ключевые слова и фразы: ближайшая по энтропии мартингальная мера, внутренняя энергия, давление, информация КульбакаЛейблера, количество информации, масса, метастабильное состояние, объем, относительная энтропия, плотность, преобразование Эшера, равновесное состояние, распределение Гиббса, свободная энергия, сжатие данных, средняя стоимость кодирования, температура, теорема Нернста, экспоненциальная полезность, энтропия.

\section{1. Введение}

1.1. Теория вероятностей: минимум и критические точки относительной энтропии. Пусть $(\Omega, \mathscr{F}, \mathbf{P})$ - измеримое пространство с $\sigma$-конечной мерой $\mathbf{P}$. Пусть $X: \Omega \rightarrow \mathbf{R}^{d}-$ измеримая функция. Рассмотрим проблему минимизации относительной энтропии (известной также как информация Кульбака-Лейблера)

$$
H(\mathbf{Q}, \mathbf{P})= \begin{cases}\int_{\Omega} \ln \frac{d \mathbf{Q}}{d \mathbf{P}} d \mathbf{Q}, & \text { если } \quad \int_{\Omega}\left|\ln \frac{d \mathbf{Q}}{d \mathbf{P}}\right| d \mathbf{Q}<\infty, \\ +\infty, & \text { если } \quad \int_{\Omega}\left|\ln \frac{d \mathbf{Q}}{d \mathbf{P}}\right| d \mathbf{Q}=\infty\end{cases}
$$

* Московский государственный университет им. М. В. Ломоносова, физический факультет, кафедра квантовой статистики и теории поля, Ленинские горы, 119992 Москва, Россия; e-mail: viktor_maslov@hotmail.com

** Московский государственный университет им. М.В. Ломоносова, механикоматематический факультет, кафедра теории вероятностей, Ленинские горы, 119992 Москва, Россия; e-mail: cherny@mech.math.msu.su 
по множеству

$$
\begin{aligned}
\mathscr{E}=\{\mathbf{Q}: \mathbf{Q}-\text { вероятностная мера на }(\Omega, \mathscr{F}) \\
\left.\mathbf{Q} \ll \mathbf{P}, \int_{\Omega}\|X\| d \mathbf{Q}<\infty, \int_{\Omega} X d \mathbf{Q}=0\right\} .
\end{aligned}
$$

Эта проблема (а также более общая проблема, когда имеется бесконечно много ограничений вида $\int_{\Omega} X^{i} d \mathbf{Q}=0$ ) хорошо известна в теории вероятностей; см. [1, с. 30], [2, гл. 11], [3], [6, §3.2], [7]. Доказываемая ниже теорема 2.1 следует из [3, теорема 3.1$]$ в том случае, когда мера $\mathbf{P}$ конечна. Однако мы приводим прямое доказательство по двум причинам: во-первых, результат для бесконечной меры $\mathbf{P}$ не выводится непосредственно из [3] (рассмотрение этого случая необходимо нам для применений в статистической физике и теории информации); во-вторых, доказательство в [3] не использует метод множителей Лагранжа, в то время как мы отдаем предпочтение этому методу с целью подчеркнуть связь рассматриваемой проблемы со статистической физикой.

Мера, на которой функционал $\mathbf{Q} \mapsto H(\mathbf{Q}, \mathbf{P})$ принимает минимальное значение, является его критической точкой. Представляется интересным найти и другие критические точки. В теореме 2.2 мы описываем все критические точки этого функционала на множестве $\mathscr{E}$.

Проблема минимизации относительной энтропии при линейных ограничениях возникает в следующих дисциплинах:

- теория больших уклонений,

- математическая статистика,

- статистическая физика,

- теория информации,

- финансовая математика.

В теории больших уклонений эта проблема возникает в теореме Санова и теореме об условном пределе (см. [2, гл. 12]). В математической статистике эта проблема возникает при доказательстве леммы Стейна и теоремы Чернова (см. [2, гл. 12]). Связь с другими тремя дисциплинами описана ниже.

1.2. Статистическая физика: равновесные и метастабильные состояния. В статистической физике проблема минимизации относительной энтропии при линейных ограничениях соответствует максимизации (больцмановской) энтропии системы при фиксированной внутренней энергии (энтропия в физике рассматривается с противоположным знаком по отношению к относительной энтропии в теории вероятностей), т.е. нахождению равновесного состояния системы; см. [11] (см. также [10]). В разделе 3 результаты раздела 2 переведены на физический язык, что дает формулы для распределения Гиббса, свободной энергии, внутренней энергии и энтропии системы, находящейся в равновесном 
состоянии, а также основные термодинамические соотношения между этими величинами (см. теорему 3.1).

Проблема нахождения критических точек функционала

$$
\mathbf{Q} \mapsto H(\mathbf{Q}, \mathbf{P})
$$

на множестве $\mathscr{E}$ соответствует нахождению метастабильных состояний системы; см. [12].

1.3. Теория информации: сжатие данных. В теории информации проблема минимизации относительной энтропии при линейных ограничениях соответствует максимизации количества информаиии, содержащегося в кодировании при фиксированной средней стоимости кодирования. Эта проблема была рассмотрена Р.Л. Стратоновичем [17] под названием «первая вариационная задача». Из ее решения вытекает, в частности, что максимальное количество информации, которое может содержаться в словах $D$-ичного алфавита со средней длиной $L$, приблизительно равно $L \ln D$ при большом $L$. Обычно этот результат выводится из совершенно других соображений (неравенство Макмиллана); см. [2, гл. 5]. Теоретико-информационная интерпретация рассматриваемой проблемы приведена в разделе 4.

\section{4. Финансовая математика: ближайшая по энтропии мар-} тингальная мера. В финансовой математике проблема минимизации относительной энтропии при линейных ограничениях соответствует нахождению ближайшей по энтропии мартингальной меры в одношаговой модели эволюции цен. В случае, когда случайные величины, описывающие цены активов, имеют экспоненциальные моменты, ее решение хорошо известно (см., например, [6, следствие 3.27]). Именно, ближайшая по энтропии мартингальная мера является преобразованием Эшера исходной вероятностной меры $\mathbf{P}$.

В многошаговой модели и в модели непрерывного времени явный вид ближайшей по энтропии мартингальной меры известен в некоторых частных случаях; см. [8], [13]. В разделе 5 мы приводим явный вид ближайшей по энтропии мартингальной меры в общей модели с дискретным временем при естественных ограничениях интегрируемости (см. теорему 5.2).

Как отмечено в ряде работ, проблема нахождения ближайшей по энтропии мартингальной меры двойственна проблеме максимизации экспоненииальной полезности; см. [4], [7], [9], [15]. В разделе 5 мы также приводим явное решение задачи максимизации экспоненциальной полезности в общей модели с дискретным временем при естественных ограничениях интегрируемости (см. теорему 5.3). Двойственность двух рассматриваемых проблем становится очевидной из сравнения их решений. 


\section{2. Теория вероятностей: минимум и критические точки относительной энтропии}

\section{1. Минимизация относительной энтропии при линейных} ограничениях. Пусть $(\Omega, \mathscr{F}, \mathbf{P})$ - измеримое пространство с $\sigma$-конечной мерой $\mathbf{P}$. Пусть $X: \Omega \rightarrow \mathbf{R}^{d}-$ измеримая функция. Найдем минимальное значение функционала $\mathbf{Q} \mapsto H(\mathbf{Q}, \mathbf{P})$, определенного в (1.1), по множеству $\mathscr{E}$, определенному в (1.2). Применим метод множителей Лагранжа и прежде всего найдем минимум выражения $\int_{\Omega}\langle\tau, X\rangle d \mathbf{Q}+H(\mathbf{Q}, \mathbf{P})$ по всем вероятностным мерам $\mathbf{Q} \ll \mathbf{P}$ (через $\langle\cdot, \cdot\rangle$ мы обозначаем скалярное произведение в $\mathbf{R}^{d}$ ).

Лемма 2.1. Пусть $Z: \Omega \rightarrow \mathbf{R}-$ случайная величина. Предположим, ито $\int_{\Omega} e^{Z} d \mathbf{P}<\infty u \int_{\Omega}|Z| e^{Z} d \mathbf{P}<\infty$. Тогда минимум выражения $-\int_{\Omega} Z d \mathbf{Q}+H(\mathbf{Q}, \mathbf{P})$ по всем вероятностным мерам $\mathbf{Q} \ll \mathbf{P}$ $c \int_{\Omega}|Z| d \mathbf{Q}<\infty$ достигается на единственной мере

$$
\mathbf{Q}_{*}=\text { const } e^{Z} \mathbf{P} \text {. }
$$

Д о к а за т е л ь с т в о. Для любой меры $\mathbf{Q}$ с указанными свойствами имеем

$$
\begin{aligned}
H\left(\mathbf{Q}, \mathbf{Q}_{*}\right) & =\int_{\Omega} \ln \frac{d \mathbf{Q}}{d \mathbf{Q}_{*}} d \mathbf{Q}=\int_{\Omega} q \ln \left(q e^{-Z} \int_{\Omega} e^{Z} d \mathbf{P}\right) d \mathbf{P} \\
& =-\int_{\Omega} Z q d \mathbf{P}+\int_{\Omega} q \ln q d \mathbf{P}+\ln \int_{\Omega} e^{Z} d \mathbf{P} \\
& =-\int_{\Omega} Z d \mathbf{Q}+H(\mathbf{Q}, \mathbf{P})+\ln \int_{\Omega} e^{Z} d \mathbf{P}
\end{aligned}
$$

где $q=\frac{d \mathbf{Q}}{d \mathbf{P}}$. Отсюда вытекает требуемое утверждение.

Положим $S=\operatorname{supp}\left(\mathbf{P} \circ X^{-1}\right)$. Пусть $L$ обозначает наименьшее аффинное подпространство $\mathbf{R}^{d}$, содержащее $S$. Через $\stackrel{\circ}{S}$ обозначим относительную внутренность $S$, т.е. внутренность $S$ в относительной топологии $L$. Если $0 \in \stackrel{\circ}{S}$, то $\mathscr{E} \neq \varnothing$ (см. [16, гл. V, §2e]).

Рассмотрим функцию

$$
\varphi(\tau)=\ln \int_{\Omega} e^{\langle\tau, X\rangle} d \mathbf{P}, \quad \tau \in \mathbf{R}^{d},
$$

принимающую значения в $(-\infty, \infty]$. Из неравенства Йенсена следует, что $\varphi$ выпукла.

Нам также понадобится функция

$$
\psi(\tau)=\frac{\int_{\Omega} X e^{\langle\tau, X\rangle} d \mathbf{P}}{\int_{\Omega} e^{\langle\tau, X\rangle} d \mathbf{P}},
$$


определенная на множестве

$$
\left\{\tau \in \mathbf{R}^{d}: \int_{\Omega}\|X\| e^{\langle\tau, X\rangle} d \mathbf{P}<\infty, \int_{\Omega} e^{\langle\tau, X\rangle} d \mathbf{P}<\infty\right\}
$$

Заметим, что для $\tau$ из внутренности множества $\left\{\tau \in \mathbf{R}^{d}: \varphi(\tau)<\infty\right\}$ имеем $\psi(\tau)=\operatorname{grad} \varphi(\tau)$.

Теорема 2.1. Предположим, что $0 \in \stackrel{\circ}{S}$.

(i) имеем

$$
\inf \{H(\mathbf{Q}, \mathbf{P}): \mathbf{Q} \in \mathscr{E}\}=-\inf \left\{\varphi(\tau): \tau \in \mathbf{R}^{d}\right\}
$$

(ii) Ecли существует $\tau_{*} \in \mathbf{R}^{d}$ maкое, ито $\varphi\left(\tau_{*}\right)=\inf \left\{\varphi(\tau): \tau \in \mathbf{R}^{d}\right\}$ $u \psi\left(\tau_{*}\right)=0$, то минимум $H(\mathbf{Q}, \mathbf{P})$ по множеству $\mathscr{E}$ достигается на единственной мере

$$
\mathbf{Q}_{*}=\text { const } e^{\left\langle\tau_{*}, X\right\rangle} \mathbf{P} \text {. }
$$

В противном случае минимум $H(\mathbf{Q}, \mathbf{P})$ по множеству $\mathscr{E}$ не достигаemcs.

З а м е ч а н и е. Имеем $\varphi(\tau)=\varphi\left(\operatorname{pr}_{L} \tau\right), \psi(\tau)=\psi\left(\operatorname{pr}_{L} \tau\right)$, где $\operatorname{pr}_{L}$ обозначает ортогональную проекцию на $L$ (условие $0 \in \stackrel{\circ}{S}$ обеспечивает, что $L$ является не только аффинным, но также и линейным подпространством). Функция $\varphi$ строго выпукла на $L$. Поэтому множество точек $\tau_{*}$, удовлетворяющих условиям $\varphi\left(\tau_{*}\right)=\inf \left\{\varphi(\tau): \tau \in \mathbf{R}^{d}\right\}$ и $\psi\left(\tau_{*}\right)=0$, либо пусто, либо имеет вид $\tau_{0}^{*}+L^{\perp}$, где $L^{\perp}-$ ортогональное дополнение к $L$. Итак, $\tau_{*}$ определено неоднозначно, но мера $\mathbf{Q}_{*}$ тем не менее единственна.

Д ок а з а т е л с т в о. (i) Докажем сначала неравенство

$$
\inf \{H(\mathbf{Q}, \mathbf{P}): \mathbf{Q} \in \mathscr{E}\} \leqslant-\inf \left\{\varphi(\tau): \tau \in \mathbf{R}^{d}\right\}
$$

Рассмотрим множества $A_{n} \in \mathscr{F}$ такие, что $A_{n} \subseteq A_{n+1}, 0<\mathbf{P}\left(A_{n}\right)<\infty$ и $\bigcup A_{n}=\Omega$. Положим $B_{n}=A_{n} \cap\{\|X\| \leqslant n\}, \mathbf{P}_{n}=\mathbf{P}\left(\cdot \cap B_{n}\right)$ и

$$
\varphi_{n}(\tau)=\ln \int_{\Omega} e^{\langle\tau, X\rangle} d \mathbf{P}_{n}, \quad \tau \in \mathbf{R}^{d}
$$

Имеем $\varphi_{n} \leqslant \varphi_{n+1}$, причем функции $\varphi_{n}$ стремятся к $\varphi$ поточечно. Кроме того, $\varphi(\tau)=\varphi\left(\operatorname{pr}_{L} \tau\right), \varphi_{n}(\tau)=\varphi_{n}\left(\operatorname{pr}_{L} \tau\right)$, функция $\varphi$ строго выпукла на $L$ и $\varphi(\tau) \rightarrow \infty$ на $L$ при $\|\tau\| \rightarrow \infty$. Следовательно, при всех достаточно больших $n$ существует точка $\tau_{n}^{*} \in \mathbf{R}^{d}$, в которой достигается минимум функции $\varphi_{n}$. Для меры $\mathbf{Q}_{n}^{*}=$ const $e^{\left\langle\tau_{n}^{*}, X\right\rangle} \mathbf{P}_{n}$ имеем

$$
\int_{\Omega} X d \mathbf{Q}_{n}^{*}=\frac{\int_{\Omega} X e^{\left\langle\tau_{n}^{*}, X\right\rangle} d \mathbf{P}_{n}}{\int_{\Omega} e^{\left\langle\tau_{n}^{*}, X\right)} d \mathbf{P}_{n}}=\operatorname{grad} \varphi_{n}\left(\tau_{n}^{*}\right)=0 .
$$


Следовательно, $\mathbf{Q}_{n}^{*} \in \mathscr{E}$. Далее,

$$
\begin{aligned}
H\left(\mathbf{Q}_{n}^{*}, \mathbf{P}\right) & =\int_{\Omega} \ln \frac{d \mathbf{Q}_{n}^{*}}{d \mathbf{P}} d \mathbf{Q}_{n}^{*}=\int_{\Omega} \ln \frac{d \mathbf{Q}_{n}^{*}}{d \mathbf{P}_{n}} d \mathbf{Q}_{n}^{*} \\
& =\int_{\Omega}\left\langle\tau_{n}^{*}, X\right\rangle d \mathbf{Q}_{n}^{*}-\ln \int_{\Omega} e^{\left\langle\tau_{n}^{*}, X\right\rangle} d \mathbf{P}_{n} \\
& =-\varphi_{n}\left(\tau_{n}^{*}\right) \underset{n \rightarrow \infty}{\longrightarrow}-\inf \left\{\varphi(\tau): \tau \in \mathbf{R}^{d}\right\},
\end{aligned}
$$

что доказывает (2.2).

Проверим теперь неравенство

$$
\inf \{H(\mathbf{Q}, \mathbf{P}): \mathbf{Q} \in \mathscr{E}\} \geqslant-\inf \left\{\varphi(\tau): \tau \in \mathbf{R}^{d}\right\} .
$$

Предположим противное, т.е. что существуют мера $\mathbf{Q}_{0} \in \mathscr{E}$ и точка $\tau_{0} \in \mathbf{R}^{d}$ такие, что $H\left(\mathbf{Q}_{0}, \mathbf{P}\right)<-\varphi\left(\tau_{0}\right)$. Рассмотрим множества $A_{n} \in \mathscr{F}$ такие, что $A_{n} \subseteq A_{n+1}, 0<\mathbf{P}\left(A_{n}\right)<\infty$ и $\bigcup A_{n}=\Omega$. Положим $B_{n}=$ $A_{n} \cap\{\|X\| \leqslant n\}, \mathbf{P}_{n}=\mathbf{P}\left(\cdot \cap B_{n}\right), \mathbf{Q}_{n}=\mathbf{Q}_{0}\left(\cdot \mid B_{n}\right)$ и определим $\varphi_{n}$ равенством (2.3). Поскольку

$$
\begin{aligned}
& \int_{\Omega} X d \mathbf{Q}_{n} \underset{n \rightarrow \infty}{\longrightarrow} \int_{\Omega} X d \mathbf{Q}_{0}=0, \\
& H\left(\mathbf{Q}_{n}, \mathbf{P}_{n}\right) \underset{n \rightarrow \infty}{\longrightarrow} H\left(\mathbf{Q}_{0}, \mathbf{P}\right), \quad \varphi_{n}\left(\tau_{0}\right) \underset{n \rightarrow \infty}{\longrightarrow} \varphi\left(\tau_{0}\right),
\end{aligned}
$$

то существует $n$ такое, что $-\int_{\Omega}\left\langle\tau_{0}, X\right\rangle d \mathbf{Q}_{n}+H\left(\mathbf{Q}_{n}, \mathbf{P}_{n}\right)<-\varphi_{n}\left(\tau_{0}\right)$. С другой стороны, из леммы 2.1 вытекает, что

$$
\begin{aligned}
& -\int_{\Omega}\left\langle\tau_{0}, X\right\rangle d \mathbf{Q}_{n}+H\left(\mathbf{Q}_{n}, \mathbf{P}_{n}\right) \\
& \quad \geqslant-\frac{\int_{\Omega}\left\langle\tau_{0}, X\right\rangle e^{\left\langle\tau_{0}, X\right\rangle} d \mathbf{P}_{n}}{\int_{\Omega} e^{\left\langle\tau_{0}, X\right\rangle} d \mathbf{P}_{n}}+\int_{\Omega} \frac{e^{\left\langle\tau_{0}, X\right\rangle}}{\int_{\Omega} e^{\left\langle\tau_{0}, X\right\rangle} d \mathbf{P}_{n}} \ln \frac{e^{\left\langle\tau_{0}, X\right\rangle}}{\int_{\Omega} e^{\left\langle\tau_{0}, X\right\rangle} d \mathbf{P}_{n}} d \mathbf{P}_{n} \\
& \quad=-\ln \int_{\Omega} e^{\left\langle\tau_{0}, X\right\rangle} d \mathbf{P}_{n}=-\varphi_{n}\left(\tau_{0}\right) .
\end{aligned}
$$

Полученное противоречие доказывает (2.4).

(ii) Первое утверждение непосредственно вытекает из леммы 2.1.

Предположим теперь, что не существует точки $\tau_{*} \in \mathbf{R}^{d}$ такой, что $\varphi\left(\tau_{*}\right)=\inf \left\{\varphi(\tau): \tau \in \mathbf{R}^{d}\right\}$ и $\psi\left(\tau_{*}\right)=0$. Допустим, что при этом существует мера $\mathbf{Q}_{0} \in \mathscr{E}$, на которой достигается минимум $H(\mathbf{Q}, \mathbf{P})$ по множеству $\mathscr{E}$. Обозначим $\frac{d \mathbf{Q}_{0}}{d \mathbf{P}}$ через $q_{0}$. Докажем сначала, что существуют $\tau_{0} \in \mathbf{R}^{d}$ и $c_{0} \in \mathbf{R}$ такие, что $\ln q_{0}=\left\langle\tau_{0}, X\right\rangle+c_{0} \mathbf{P}$-п.в. на множестве $\left\{q_{0}>0\right\}$. Если это не так, то найдется множество $B \in \mathscr{F}$ такое, что $0<\mathbf{P}(B)<\infty$, функция $X$ ограничена на $B, \ln q_{0}$ ограничен на $B$ и сужение $\left.\ln q_{0}\right|_{B}$ не принадлежит пространству $\mathscr{L}=\left\{\left.\langle\tau, X\rangle\right|_{B}+c\right.$ : $\left.\tau \in \mathbf{R}^{d}, c \in \mathbf{R}\right\}$. Пусть $p$ обозначает $L^{2}\left(B,\left.\mathscr{F}\right|_{B},\left.\mathbf{P}\right|_{B}\right)$-проекцию $\left.\ln q_{0}\right|_{B}$ 
на $\mathscr{L}$. Тогда функция $r=\left.\ln q_{0}\right|_{B}-p$ измерима, ограничена и удовлетворяет условиям

$$
\int_{B}|r| d \mathbf{P}<\infty, \quad \int_{B} r d \mathbf{P}=0, \quad \int_{B}|r| X d \mathbf{P}<\infty, \quad \int_{B} r X d \mathbf{P}=0 .
$$

Поскольку $\ln q_{0}$ ограничен на $B$, то $\mathbf{Q}_{0}+\varepsilon r \mathbf{P}$ является вероятностной мерой при всех $\varepsilon$, принадлежащих достаточно малой окрестности нуля. Из (2.5) следует, что $\mathbf{Q}_{0}+\varepsilon r \mathbf{P} \in \mathscr{E}$. Кроме того,

$$
\left.\frac{d}{d \varepsilon}\right|_{\varepsilon=0} H\left(\mathbf{Q}_{0}+\varepsilon r \mathbf{P}, \mathbf{P}\right)=\int_{\Omega} r\left(1+\ln q_{0}\right) d \mathbf{P}=\int_{B} r \ln q_{0} d \mathbf{P} \neq 0 .
$$

Но это противоречит выбору $\mathbf{Q}_{\mathbf{0}}$.

Итак, мы доказали, что $\mathrm{Q}_{0}$ имеет вид $\mathrm{Q}_{0}=\operatorname{const} e^{\left\langle\tau_{0}, X\right\rangle} I_{A} \mathbf{P}$ с $\tau_{0} \in \mathbf{R}^{d}, A \in \mathscr{F}$. Докажем, что $\mathbf{P}(\Omega \backslash A)=0$. Если это не так, то найдется множество $B \in \mathscr{F}$ такое, что $0<\mathbf{P}(B)<\infty$, функция $X$ ограничена на $B$ и $\mathbf{P}(B \backslash A)>0$. Из первого утверждения п. (ii) вытекает существование $\tau_{1} \in \mathbf{R}^{d}$ такого, что мера $\mathbf{Q}_{1}=$ const $e^{\left\langle\tau_{1}, X\right\rangle} I_{B} \mathbf{P}$ принадлежит $\mathscr{E}$. Тогда $(1-\varepsilon) \mathbf{Q}_{0}+\varepsilon \mathbf{Q}_{1} \in \mathscr{E}$ для любого $\varepsilon \in[0,1]$. Имеем

$$
\begin{aligned}
& H\left((1-\varepsilon) \mathbf{Q}_{0}+\varepsilon \mathbf{Q}_{1}, \mathbf{P}\right) \\
& \quad=\int_{A}\left((1-\varepsilon) q_{0}+\varepsilon q_{1}\right) \ln \left((1-\varepsilon) q_{0}+\varepsilon q_{1}\right) d \mathbf{P}+\int_{B \backslash A} \varepsilon q_{1} \ln \varepsilon q_{1} d \mathbf{P},
\end{aligned}
$$

где $q_{1}=\frac{d \mathbf{Q}_{1}}{d \mathbf{P}}$. Очевидно, найдется достаточно малое $\varepsilon>0$ такое, что $H\left((1-\varepsilon) \mathbf{Q}_{0}+\varepsilon \mathbf{Q}_{1}, \mathbf{P}\right)<H\left(\mathbf{Q}_{0}, \mathbf{P}\right)$. Но это противоречит выбору $\mathbf{Q}_{0}$.

Итак, мы доказали, что $\mathbf{Q}_{0}$ имеет вид $\mathbf{Q}_{0}=$ const $e^{\left\langle\tau_{0}, X\right\rangle} \mathbf{P}$ с $\tau_{0} \in \mathbf{R}^{d}$. Тогда

$$
\begin{aligned}
& \varphi\left(\tau_{0}\right)=-\ln \int_{\Omega} e^{\left\langle\tau_{0}, X\right\rangle} d \mathbf{P}=-H\left(\mathbf{Q}_{0}, \mathbf{P}\right), \\
& \psi\left(\tau_{0}\right)=\frac{\int_{\Omega} X e^{\left\langle\tau_{0}, X\right\rangle} d \mathbf{P}}{\int_{\Omega} e^{\left\langle\tau_{0}, X\right\rangle} d \mathbf{P}}=\int_{\Omega} X d \mathbf{Q}_{0}=0 .
\end{aligned}
$$

Если существует точка $\tau_{1} \in \mathbf{R}^{d}$ такая, что $\varphi\left(\tau_{1}\right)<\varphi\left(\tau_{0}\right)$, то

$$
\frac{\int_{\Omega}\left\langle\tau_{1}-\tau_{0}, X\right\rangle e^{\left\langle\tau_{0}, X\right\rangle} d \mathbf{P}}{\int_{\Omega} e^{\left\langle\tau_{0}, X\right\rangle} d \mathbf{P}}=\left.\frac{d}{d \varepsilon}\right|_{\varepsilon=0} \varphi\left((1-\varepsilon) \tau_{0}+\varepsilon \tau_{1}\right)<0,
$$

что противоречит (2.7). Следовательно, $\varphi\left(\tau_{0}\right)=\inf \left\{\varphi(\tau): \tau \in \mathbf{R}^{d}\right\}$. Полученное противоречие показывает, что мера $\mathbf{Q}_{0}$ не существует.

Следствие 2.1. Пусть $m \in \mathbf{R}, \sigma \neq 0$. Минимум $\int_{\mathbf{R}} q(x) \ln q(x) d x$ по множеству

$$
\left\{q \geqslant 0: \int_{\mathbf{R}} q(x) d x=1, \int_{\mathbf{R}} x q(x) d x=m, \int_{\mathbf{R}}(x-m)^{2} q(x) d x=\sigma^{2}\right\}
$$


достигается (только) на плотности нормального распределения со средним $m$ u дисперсией $\sigma^{2}$.

Следствие 2.2. Пусть $m>0$. Минимум $\int_{\mathbf{R}_{+}} q(x) \ln q(x) d x$ по множеству

$$
\left\{q \geqslant 0: \int_{\mathbf{R}_{+}} q(x) d x=1, \int_{\mathbf{R}_{+}} x q(x) d x=m\right\}
$$

достигается (только) на плотности экспоненциального распределения со средним $m$.

2.2. Критические точки относительной энтропии. Мы нашли меру, на которой достигается минимальное значение функционала $\mathbf{Q} \mapsto H(\mathbf{Q}, \mathbf{P})$ по множеству $\mathscr{E}$. Найдем теперь критические точки этого функционала. Для $\mathbf{Q} \in \mathscr{E}$ обозначим через $\mathscr{D}_{\mathbf{Q}}$ множество измеримых ограниченных функций $r: \Omega \rightarrow \mathbf{R}$ со следующим свойством: сушествует $\delta>0$ такое, что $\mathbf{Q}+r \varepsilon \mathbf{P} \in \mathscr{E}$ для любого $\varepsilon \in[0, \delta)$.

О п р е д е ле н и е 2.1. Элемент $\mathbf{Q} \in \mathscr{E}$ назовем критической точкой функционала $\mathbf{Q} \mapsto H(\mathbf{Q}, \mathbf{P})$, если для любого $r \in \mathscr{D}_{\mathbf{Q}}$ производная $\left.\frac{d}{d \varepsilon}\right|_{\varepsilon=0} H(\mathbf{Q}+\varepsilon r \mathbf{P}, \mathbf{P})$ либо не существует, либо равна нулю.

Теорема 2.2. Элемент $\mathbf{Q} \in \mathscr{E}$ является критической точкой функиионала $\mathbf{Q} \mapsto H(\mathbf{Q}, \mathbf{P})$ тогда и только тогда, когда $\mathbf{Q}$ имеет вид

$$
\mathbf{Q}=\text { const } e^{\langle\tau, X\rangle} I_{A} \mathbf{P}, \quad \text { zдe } \quad \tau \in \mathbf{R}^{d}, A \in \mathscr{F} .
$$

Д о к а з а т е л ь с т в о. Предположим, что $\mathbf{Q}$ имеет указанный вид. Тогда для любого $r \in \mathscr{D}_{\mathbf{Q}}$ имеем

$$
H(\mathbf{Q}+\varepsilon r \mathbf{P}, \mathbf{P})=\int_{\Omega}(q+\varepsilon r) \ln (q+\varepsilon r) d \mathbf{P},
$$

где $q=\frac{d \mathbf{Q}}{d \mathbf{P}}$. Если $\mathbf{P}\{r>0, q=0\}=0$, то

$$
\begin{aligned}
\left.\frac{d}{d \varepsilon}\right|_{\varepsilon=0} H(\mathbf{Q}+\varepsilon r \mathbf{P}, \mathbf{P}) & =\int_{\Omega} r(1+\ln q) d \mathbf{P}=\int_{A} r \ln q d \mathbf{P} \\
& =\int_{A} r\langle\tau, X\rangle d \mathbf{P}-\int_{A} r d \mathbf{P} \ln \int_{A} e^{\langle\tau, X\rangle} d \mathbf{P}=0 .
\end{aligned}
$$

Если $\mathbf{P}\{r>0, q=0\}>0$, то эта производная не существует.

Обратная импликация была проверена при доказательстве теоремы 2.1 (ii).

\section{3. Статистическая физика: равновесные и метастабильные состояния}

3.1. Равновесные состояния. Рассмотрим систему, состояшую из большого числа частиц. Каждая из частиц может находиться в одном из элементарных состояний $\omega \in \Omega$. Состоянием системы назовем 
неотрицательную конечную меру $\mathbf{Q}$ такую, что $\mathbf{Q} \ll \mathbf{P}$, где $\mathbf{P}-$ фиксированная неотрицательная $\sigma$-конечная мера на $\Omega$. Значение $\mathbf{Q}(\Omega)$ интерпретируется как масса системы, а значение $\mathbf{P}(\Omega)$ интерпретируется как объем системы. Если система находится в состоянии $\mathbf{Q}$, то для любого измеримого множества $A \subseteq \Omega$ полная масса частиц, находящихся в элементарных состояниях из $A$, есть $\mathbf{Q}(A)$. Пусть $X: \Omega \rightarrow \mathbf{R}_{+}$- некоторая функция, значение которой в точке $\omega$ интерпретируется как энергетический уровень, соответствуюший $\omega$. Внутренняя энергия системы, находящейся в состоянии $\mathbf{Q}$, есть $\int_{\Omega} X d \mathbf{Q}$. (Больцмановская) энтропия системы, находящейся в состоянии $\mathbf{Q}$, есть $-\int_{\Omega} \ln \left(\frac{1}{\mathbf{Q}(\Omega)} \frac{d \mathbf{Q}}{d \mathbf{P}}\right) d \mathbf{Q}$ (заметим, что для вероятностных мер $\mathbf{Q}$ эта величина совпадает с $H(\mathbf{Q}, \mathbf{P})$ с точностью до знака).

В этой интерпретации проблема минимизации относительной энтропии при линейных ограничениях соответствует максимизации энтропии при фиксированной внутренней энергии (ограничение $\int_{\Omega} X d \mathbf{Q}=0$ следует заменить ограничением $\int_{\Omega} X d \mathbf{Q}=E$, где $E$ - положительное число). Проблема нахождения меры $\mathbf{Q}_{*}$, на которой достигается минимум $H(\mathbf{Q}, \mathbf{P})$ (по множеству мер $\mathbf{Q} \ll \mathbf{P}$, удовлетворяющих ограничениям $\mathbf{Q}(\Omega)=M$ и $\left.\int_{\Omega} X d \mathbf{Q}=E\right)$, соответствует нахождению равновесного состояния системы при фиксированной массе $M$ и внутренней энергии $E$.

Величина

$$
\int_{\Omega} X d \mathbf{Q}+T \int_{\Omega} \ln \left(\frac{1}{\mathbf{Q}(\Omega)} \frac{d \mathbf{Q}}{d \mathbf{P}}\right) d \mathbf{Q}
$$

интерпретируется как сөободная энергия системы, находящейся в состоянии $\mathbf{Q}$, а множитель Лагранжа $T>0$ интерпретируется как темпераmypa.

В этой интерпретации проблема минимизации выражения (3.1) (см. лемму 2.1) соответствует минимизации свободной энергии при фиксированной температуре. Проблема нахождения меры $\mathbf{Q}_{*}$, на которой достигается минимум выражения (3.1) (по всем мерам $\mathbf{Q} \ll \mathbf{P}$ с $\mathbf{Q}(\Omega)=M$ ), соответствует нахождению равновесного состояния системы при фиксированной массе $M$ и температуре $T$.

Приводимое ниже утверждение следует из леммы 2.1. Оно дает равновесное состояние, свободную энергию, внутреннюю энергию и энтропию равновесного состояния.

Теорема 3.1. Пусть $M>0, T>0$. Предположим, что $\int_{\Omega} e^{-X / T} d \mathbf{P}<\infty u \int_{\Omega} X e^{-X / T} d \mathbf{P}<\infty$.

(i) Минимум выражения (3.1) по неотричательньм мерам $\mathbf{Q} \ll \mathbf{P}$ c $\mathbf{Q}(\Omega)=M$ достигается на единственной мере

$$
\mathbf{Q}_{*}=M \frac{e^{-X / T}}{\int_{\Omega} e^{-X / T} d \mathbf{P}} \mathbf{P} .
$$


(ii) Обозначим через $F, E$ u $S$ свободную энергию, внутреннюю энергию $и$ энтропию состояния $\mathbf{Q}_{*}$. Тогда

$$
\begin{aligned}
& F=-M T \ln \left(\int_{\Omega} e^{-X / T} d \mathbf{P}\right), \quad E=M \frac{\int_{\Omega} X e^{-X / T} d \mathbf{P}}{\int_{\Omega} e^{-X / T} d \mathbf{P}} \\
& S=\frac{M}{T} \frac{\int_{\Omega} X e^{-X / T} d \mathbf{P}}{\int_{\Omega} e^{-X / T} d \mathbf{P}}+M \ln \left(\int_{\Omega} e^{-X / T} d \mathbf{P}\right) .
\end{aligned}
$$

(iii) Предположим, ито мера $\mathbf{P}$ конечна, т.е. $\mathbf{P}=V \mathbf{P}_{0}$, где $\mathbf{P}_{0}$ вероятностная мера. Тогда

$$
\frac{\partial F}{\partial V}=-p, \quad \frac{\partial E}{\partial V}=0, \quad \frac{\partial S}{\partial V}=\rho, \quad \frac{\partial F}{\partial T}=-S, \quad \frac{\partial E}{\partial T}=T \frac{\partial S}{\partial T},
$$

где $p:=M T / V$ интерпретируется как давление, а $\rho:=M / V$ интерпретируется как плотность.

(iv) Ecли мера $\mathbf{P} \circ X^{-1}$ невырождена, то $E$ u $S$ строго возрастают no $T$.

(v) Положим $a=\sup \{x \in \mathbf{R}: X \geqslant x$ P-п.в. $\}$. Тогдa

$$
E \underset{T \downarrow 0}{\longrightarrow} a, \quad S \underset{T \downarrow 0}{\longrightarrow} M \ln \mathbf{P}\{X=a\} .
$$

3 а м е ч а н и е. Мера $\mathbf{Q}_{*}$, определенная в (i), соответствует распределению Гиббса (см. [11]). Равенства п. (iii) выражают известные соотношения термодинамики. Утверждение п. (v) для случая, когда $\mathbf{P}-$ считающая мера, соответствует теореме Нернста (см. [11]).

3.2. Метастабильные состояния. Проблема нахождения критических точек функционала $\mathbf{Q} \mapsto H(\mathbf{Q}, \mathbf{P})$ (см. теорему 2.2 ) соответствует нахождению метастабильньх состояний системы, т.е. состояний, в которых система может находиться очень длительное время, прежде чем перейдет в равновесное состояние. Теорема 2.2 показывает, что метастабильные состояния имеют вид

$$
\mathbf{Q}_{*}^{A}=M \frac{e^{-X / T}}{\int_{A} e^{-X / T} d \mathbf{P}} I_{A} \mathbf{P},
$$

где $A \in \mathscr{F}$. Утверждения теоремы 3.1 (ii)-(v) переносятся на свободную энергию, внутреннюю энергию и энтропию $\mathbf{Q}_{*}^{A}$ заменой $\mathbf{P}$ на $\left.\mathbf{P}\right|_{A}$.

\section{4. Теория информации: сжатие данных}

Пусть $\Omega$ - некоторое множество кодовых слов ( $\Omega$ может быть конечным или счетным). Каждому кодовому слову $\omega$ соответствует его стоимость $X(\omega) \geqslant 0$. Пусть $\left(\Omega_{0}, \mathscr{F}_{0}, \mathbf{P}_{0}\right)$ - вероятностное пространство, интерпретируемое как источник информации. Кодирование есть отображение $F: \Omega_{0} \rightarrow \Omega$. Это отображение индуцирует меру $\mathbf{Q}=\mathbf{Q}_{0} \circ F^{-1}$ 
на $\Omega$. Число $\sum_{\omega \in \Omega} X(\omega) q(\omega)$ назовем средней стоимостью кодирования $F$. Число $-\sum_{\omega \in \Omega} q(\omega) \ln q(\omega)$ назовем количеством информаиии, содержащимся в кодировании $F$. Проблема сжатия данных заключается в максимизации количества информации, содержащегося в кодировании, при фиксированной средней стоимости кодирования.

Рассмотрим функцию

$$
\varphi(\tau)=\ln \sum_{\omega \in \Omega} e^{\tau X(\omega)}, \quad \tau \in \mathbf{R}
$$

(она может принимать значение $+\infty$ ). Заметим, что $\varphi$ дифференцируема внутри интервала $\{\tau \in \mathbf{R :} \varphi(\tau)<\infty\}$.

$\mathrm{B}$ дальнейшем будем использовать обозначение

$$
H(C)=\sup \left\{-\sum_{\omega \in \Omega} q(\omega) \ln q(\omega)\right\}
$$

где супремум берется по вероятностным мерам $\mathbf{Q}$ на $\Omega$, удовлетворяющим условию $\sum_{\omega \in \Omega} X(\omega) q(\omega) \leqslant C$.

Теорема 4.1. Пусть $C>0$. Предположим, ито существует $\tau_{*} \in \mathbf{R}$ maкое, чmо $\varphi^{\prime}\left(\tau_{*}\right)=C$. Тогда

$$
H(C)=\varphi\left(\tau_{*}\right)-C \tau_{*}
$$

Д оказательство. Утверждение вытекает из теоремы 2.1 (необходимо рассмотреть $X-C$ вместо $X$ и взять считающую меру в качестве $\mathbf{P}$ ).

Пример 4.1. Пусть $\Omega=\left\{\left(a_{1}, \ldots, a_{n}\right): n \in \mathbf{N}, a_{i} \in A\right\}$, где $A-$ D-ичный алфавит. Пусть стоимость слова $\left(a_{1}, \ldots, a_{n}\right)$ есть $n$. Тогда

$$
\lim _{C \rightarrow \infty} \frac{H(C)}{C}=\ln D
$$

Доказ ательство. Имеем

$$
\varphi(\tau)=\ln \sum_{n=1}^{\infty} e^{\tau n} D^{n}=\ln \frac{D e^{\tau}}{1-D e^{\tau}}=\ln \left(\frac{1}{1-D e^{\tau}}-1\right), \quad \tau \in \mathbf{R} .
$$

Функция $\varphi$ конечна на интервале $(-\infty,-\ln D)$ и

$$
\varphi^{\prime}(\tau)=\frac{D e^{\tau}\left(1-D e^{\tau}\right)}{\left(1-D e^{\tau}\right)^{2} D e^{\tau}}=\frac{1}{1-D e^{\tau}}, \quad \tau \in(-\infty,-\ln D) .
$$

Для точки $\tau_{*}=\tau_{*}(C)$, определенной как решение уравнения $\varphi^{\prime}\left(\tau_{*}\right)=C$, имеем (см. рис. 1)

$$
\lim _{C \rightarrow \infty} \frac{\varphi\left(\tau_{*}\right)-C \tau_{*}}{C}=\lim _{C \rightarrow \infty} \frac{\ln (C-1)}{C}-\lim _{C \rightarrow \infty} \tau_{*}=-\lim _{C \rightarrow \infty} \tau_{*}=\ln D .
$$




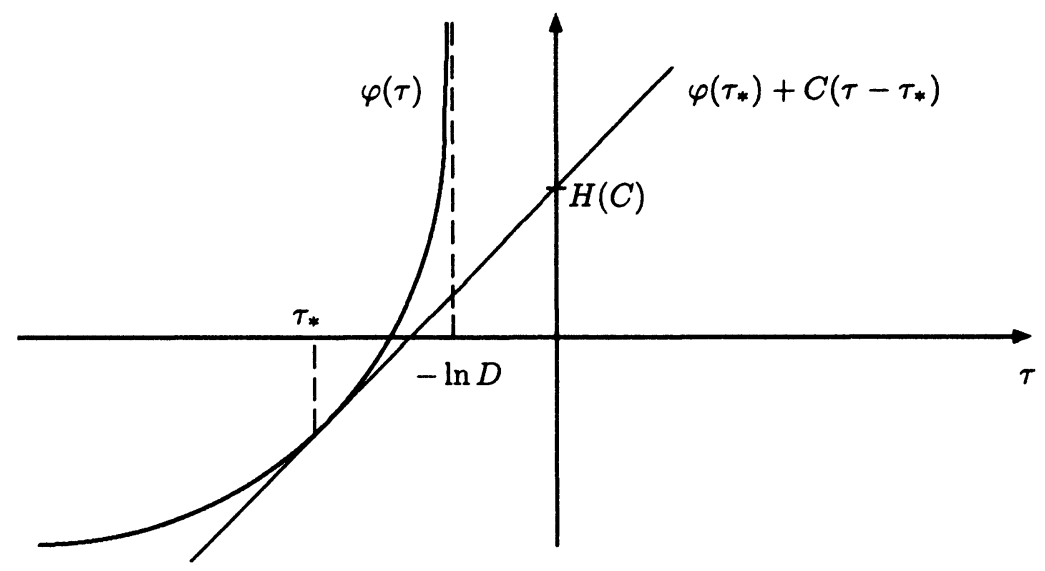

Рис. 1

Пример 4.2. Пусть $\Omega=\left\{\left(a_{1}, \ldots, a_{n}\right): n \in \mathbf{N}, a_{i} \in A\right\}$, где $A=\left\{A_{1}, \ldots, A_{D}\right\}-D$-ичный алфавит. Пусть стоимость буквы $A_{i}$ есть $\lambda\left(A_{i}\right)$, так ито стоимость кодового слова $\left(a_{1}, \ldots, a_{n}\right)$ есть $\lambda\left(a_{1}\right)+$ $\cdots+\lambda\left(a_{n}\right)$. Тогдa

$$
\lim _{C \rightarrow \infty} \frac{H(C)}{C}=-\tau_{0}
$$

где $\tau_{0}$ определяется из уравнения $\sum_{i=1}^{D} e^{\tau_{0} \lambda\left(A_{i}\right)}=1$.

Д ок а з а те л ь ств о. Имеем

$$
\begin{aligned}
\varphi(\tau) & =\ln \sum_{n=1}^{\infty} \sum_{i_{1}, \ldots, i_{n}} \exp \left\{\tau \lambda\left(A_{i_{1}}\right)+\cdots+\tau \lambda\left(A_{i_{n}}\right)\right\} \\
& =\ln \sum_{n=1}^{\infty}\left(\sum_{i=1}^{D} e^{\tau \lambda\left(A_{i}\right)}\right)^{n}=\ln \frac{\eta(\tau)}{1-\eta(\tau)}=\ln \left(\frac{1}{1-\eta(\tau)}-1\right), \quad \tau \in \mathbf{R},
\end{aligned}
$$

где $\eta(\tau)=\sum_{i=1}^{D} e^{\tau \lambda\left(A_{i}\right)}, \tau \in \mathbf{R}$. Функция $\varphi$ конечна на интервале $\left(-\infty, \tau_{0}\right)$, и

$$
\varphi^{\prime}(\tau)=\frac{\eta^{\prime}(\tau)}{\eta(\tau)(1-\eta(\tau))}, \quad \tau \in\left(-\infty, \tau_{0}\right)
$$

Для точки $\tau_{*}=\tau_{*}(C)$, определенной как решение уравнения $\varphi^{\prime}\left(\tau_{*}\right)=C$, имеем $\lim _{C \rightarrow \infty} \tau_{*}=\tau_{0}$, откуда

$$
\lim _{C \rightarrow \infty} \frac{\varphi\left(\tau_{*}\right)-C \tau_{*}}{C}=\lim _{C \rightarrow \infty} \frac{1}{C} \ln \left(\frac{C \eta\left(\tau_{*}\right)}{\eta^{\prime}\left(\tau_{*}\right)}-1\right)-\lim _{C \rightarrow \infty} \tau_{*}=-\tau_{0}
$$




\section{5. Финансовая математика: ближайшая по энтропии} мартингальная мера

\section{1. Одношаговая модель. Пусть}

$$
\left(\Omega, \mathscr{F},\left(\mathscr{F}_{n}\right)_{0 \leqslant n \leqslant N}, \mathbf{P},\left(X_{n}\right)_{0 \leqslant n \leqslant N}\right)
$$

есть модель эволюции цен нескольких финансовых активов. В этой модели $\left(\mathscr{F}_{n}\right)$ - фильтрация, а $X$ является $d$-мерным $\left(\mathscr{F}_{n}\right)$-согласованным процессом. С финансовой точки зрения, $X_{n}^{i}$ есть (дисконтированная) цена $i$-го актива в момент времени $n$. Напомним прежде всего некоторые определения и факты финансовой математики.

О п р е д е л е н и е 5.1. Самофинансируемой стратегией называется пара $\pi=(x, H)$, где $x \in \mathbf{R}$, а $H=\left(H_{n}^{i} ; n=1, \ldots, N, i=\right.$ $1, \ldots, d)-\left(\mathscr{F}_{n}\right)$-предсказуемый процесс, т.е. случайная величина $H_{n}$ $\mathscr{F}_{n-1}$-измерима для любого $n=1, \ldots, N$. Значение $x$ называется начальныцм капиталом стратегии $\pi$. (Дисконтированным) капиталом стратегии $\pi$ называется процесс

$$
X_{n}^{\pi}=x+\sum_{i=1}^{n}\left\langle H_{i}, \Delta X_{i}\right\rangle, \quad n=0, \ldots, N,
$$

где $\Delta X_{n}=X_{n}-X_{n-1}$.

О п р е д е л е н и е 5.2. Стратегия $\pi=(x, H)$ реализует арбитраж, если (i) $x=0$, (ii) $X_{N}^{\pi} \geqslant 0$ P-п.н., (iii) $\mathbf{P}\left\{X_{N}^{\pi}>0\right\}>0$.

Модель называется безарбитражной, если такой стратегии не существует.

Обозначим $\mathbf{P}_{n}(\omega)$ условное распределение $\operatorname{Law}\left(\Delta X_{n} \mid \mathscr{F}_{n-1}\right)(\omega)$ и $S_{n}(\omega)$ - носитель $\mathbf{P}_{n}(\omega)$. Пусть $L_{n}(\omega)$ - наименьшее аффинное подпространство $\mathbf{R}^{d}$, содержащее $S_{n}(\omega)$. Обозначим через $\stackrel{\circ}{S}_{n}(\omega)$ относительную внутренность $S_{n}(\omega)$, т.е. внутренность $S_{n}(\omega)$ в относительной топологии $L_{n}(\omega)$.

Предложение 5.1 (первая фундаментальная теорема теории арбитража). Следуюшие условия эквивалентны:

(i) модель является безарбитражной;

(ii) существует вероятностная мера $\mathbf{Q} \sim \mathbf{P}$ такая, что $X$ является $\left(\mathscr{F}_{n}, \mathbf{Q}\right)$-мартингалом;

(iii) для любого $n=1, \ldots, N$ и Р-п.в. $\omega$ имеем $0 \in \stackrel{\circ}{S}_{n}(\omega)$.

Доказательство можно найти, например, в $[16$, гл. V, § 2e].

Ниже мы будем использовать обозначение

$$
\begin{aligned}
\mathscr{M}^{a}=\{\mathbf{Q}: \mathbf{Q}-\text { вероятностная мера на }(\Omega, \mathscr{F}), \mathbf{Q} \ll \mathbf{P} \\
\text { и } \left.X \text { является }\left(\mathscr{F}_{n}, \mathbf{Q}\right) \text {-локальным мартингалом }\right\} .
\end{aligned}
$$

Важной задачей финансовой математики является нахождение «наиболее естественного» элемента множества $\mathscr{M}^{a}$. Один из возможных путей 
ее решения заключается в нахождении того элемента $\mathscr{M}^{a}$, на котором достигается минимум относительной энтропии $H(\mathbf{Q}, \mathbf{P})$. Соответствующая мера называется ближайшей по энтропии мартингальной мерой. Приводимый ниже результат хорошо известен. Он показывает, что в одношаговой модели ближайшая по энтропии мартингальная мера является преобразованием Эиера меры $\mathbf{P}$.

Теорема 5.1. Рассмотрим безарбитражную модель вида (5.1) $c N=1$. Предположим, что $\sigma$-алгебра $\mathscr{F}_{0}$ P-тривиальна $u$ $\mathbf{E}_{\mathbf{P}} e^{\left\langle\tau, \Delta X_{1}\right\rangle}<\infty$ для любого $\tau \in \mathbf{R}^{d}$. Тогда существует точка $\tau_{*}$, в которой достигается минимум функиии $\varphi(\tau):=\mathbf{E}_{\mathbf{P}} e^{\left\langle\tau, \Delta X_{1}\right\rangle}$. Минимум $H(\mathbf{Q}, \mathbf{P})$ по множеству $\mathscr{M}^{a}$ достигается на единственной мере

$$
\mathbf{Q}_{*}=\text { const } e^{\left\langle\tau_{*}, \Delta X_{1}\right\rangle} \mathbf{P} .
$$

Д о к а з а т е л с т в о. Заметим, что в данном случае

$$
\mathscr{M}^{a}=\left\{\mathbf{Q}: \mathbf{Q}-\text { вероятностная мера на }(\Omega, \mathscr{F}), \mathbf{Q} \ll \mathbf{P}, \mathbf{E}_{\mathbf{Q}} \Delta X_{1}=0\right\} \text {. }
$$

Теперь требуемое утверждение вытекает из теоремы 2.1.

5.2. Многошаговая модель. Наша следующая цель - описать структуру ближайшей по энтропии мартингальной меры в общей многошаговой модели. Нам потребуется вспомогательная лемма.

Лемма 5.1. Пусть $(\Omega, \mathscr{F}, \mathbf{P})$ - вероятностное пространство, $\mathscr{G} \subseteq \mathscr{F}-$ под- $\sigma$-алгебра, $Y: \Omega \rightarrow \mathbf{R}-$ случайная величина, $X: \Omega \rightarrow$ $\mathbf{R}^{d}-$ случайный вектор, причем $\mathbf{E}_{\mathbf{P}}\left(e^{(\tau, X)+Y} \mid \mathscr{G}\right)<\infty \mathbf{P}-$ n.н. для любого $\tau \in \mathbf{R}^{d}$. Предположим, ито $0 \in \stackrel{\circ}{S}(\omega)$ для $\mathbf{P}$-n.в. $\omega$, где $\stackrel{\circ}{S}(\omega)$ обозначает относительную внутренность условного распределения $\operatorname{Law}(X \mid \mathscr{G})(\omega)$. Тогда существует $\mathscr{G} \times \mathscr{B}\left(\mathbf{R}^{d}\right)$-измеримая версия функuиน

$$
\varphi(\omega, \tau)=\mathbf{E}_{\mathbf{P}}\left(e^{\langle\tau, X\rangle+Y} \mid \mathscr{G}\right)(\omega), \quad \omega \in \Omega, \quad \tau \in \mathbf{R}^{d},
$$

такая, что для любого $\omega$ отображение $\tau \mapsto \varphi(\tau, \omega)$ непрерывно $u$ $\varphi(\tau, \omega) \rightarrow \infty$ при $\|\tau\| \rightarrow \infty$. Для этой версии существует $\mathscr{G}$-измеримое отображение $\tau_{*}: \Omega \rightarrow \mathbf{R}^{d}$ такое, ито для $\mathbf{P}$-n.в. $\omega$

$$
\tau_{*}(\omega)=\underset{\tau \in \mathbf{R}^{d}}{\arg \min } \varphi(\omega, \tau) .
$$

Док аз ательство. Пусть $\mathbf{P}_{\omega}$ обозначает условное распределение $\operatorname{Law}(X, Y \mid \mathscr{G})(\omega)$. Обозначим через $\tilde{X}: \mathbf{R}^{d+1} \rightarrow \mathbf{R}^{d}$ и $\tilde{Y}: \mathbf{R}^{d+1} \rightarrow \mathbf{R}$ координатные отображения, т.е. $\widetilde{X}\left(x^{1}, \ldots, x^{d+1}\right)=$ $\left(x^{1}, \ldots, x^{d}\right), \tilde{Y}\left(x^{1}, \ldots, x^{d+1}\right)=x^{d+1}$. Тогда функция

$$
\varphi(\tau, \omega)= \begin{cases}\mathbf{E}_{\mathbf{P}_{\omega}} e^{\langle\tau, \widetilde{X}\rangle+\widetilde{Y}}, & \text { если } 0 \in \stackrel{\circ}{S}(\omega) \text { и } \mathbf{E}_{\mathbf{P}_{\omega}} e^{\langle\lambda, \widetilde{X}\rangle+\widetilde{Y}}<\infty \forall \lambda \in \mathbf{R}^{d}, \\ 0 & \text { иначе }\end{cases}
$$

удовлетворяет требуемым свойствам. 
Для доказательства существования $\tau_{*}$ рассмотрим функцию

$$
\eta(\omega)=\inf \left\{\varphi(\omega, \tau): \tau \in \mathbf{R}^{d}\right\}=\inf \left\{\varphi(\omega, \tau): \tau \in \mathbf{Q}^{d}\right\} .
$$

Эта функция $\mathscr{G}$-измерима, так что множество $\{(\omega, \tau): \varphi(\omega, \tau)=\eta(\omega)\}$ принадлежит $\mathscr{G} \times \mathscr{B}\left(\mathbf{R}^{d}\right)$. Теперь существование $\tau_{*}$ вытекает из теоремы об измеримом выборе (см. [5, теорема 8.2, с. 252]).

Рассмотрим теперь безарбитражную модель вида (5.1). Предположим, что $\mathbf{E}_{\mathbf{P}}\left(e^{\left\langle\tau, \Delta X_{n}\right\rangle} \mid \mathscr{F}_{n-1}\right)<\infty \mathbf{P}$-п.н. для любых $n=1, \ldots, N, \tau \in \mathbf{R}^{d}$ (например, условно-гауссовские модели удовлетворяют этому ограничению). Построим случайные величины $\tau_{N}^{*}, \ldots, \tau_{1}^{*}$ (спускаясь от $N$ к 1 ) равенством

$$
\tau_{n}^{*}=\underset{\tau \in \mathbf{R}^{d}}{\arg \min } \mathbf{E}\left(\exp \left\{\left\langle\tau, \Delta X_{n}\right\rangle+\sum_{i=n+1}^{N}\left\langle\tau_{i}^{*}, \Delta X_{i}\right\rangle\right\} \mid \mathscr{F}_{n-1}\right),
$$

как описано в лемме 5.1. Применимость этой леммы следует из предложения 5.1 и неравенств

$$
\begin{aligned}
& \mathbf{E}_{\mathbf{P}}\left(\exp \left\{\left\langle\tau, \Delta X_{n}\right\rangle+\sum_{i=n+1}^{N}\left\langle\tau_{i}^{*}, \Delta X_{i}\right\rangle\right\} \mid \mathscr{F}_{n-1}\right) \\
& =\mathbf{E}_{\mathbf{P}}\left(e ^ { \langle \tau , \Delta X _ { n } \rangle } \mathbf { E } _ { \mathbf { P } } \left(\operatorname { e x p } \left\{\left\langle\tau_{n+1}^{*}, \Delta X_{n+1}\right\rangle\right.\right.\right. \\
& \left.\left.\left.\quad+\sum_{i=n+2}^{N}\left\langle\tau_{i}^{*}, \Delta X_{i}\right\rangle\right\} \mid \mathscr{F}_{n}\right) \mid \mathscr{F}_{n-1}\right) \\
& \leqslant \mathbf{E}_{\mathbf{P}}\left(e^{\left\langle\tau, \Delta X_{n}\right\rangle} \mathbf{E}_{\mathbf{P}}\left(\exp \left\{\sum_{i=n+2}^{N}\left\langle\tau_{i}^{*}, \Delta X_{i}\right\rangle\right\} \mid \mathscr{F}_{n}\right) \mid \mathscr{F}_{n-1}\right) \\
& =\mathbf{E}_{\mathbf{P}}\left(\exp \left\{\left\langle\tau, \Delta X_{n}\right\rangle+\sum_{i=n+2}^{N}\left\langle\tau_{i}^{*}, \Delta X_{i}\right\rangle\right\} \mid \mathscr{F}_{n-1}\right) \\
& \leqslant \cdots \leqslant \mathbf{E}_{\mathbf{P}}\left(e^{\left\langle\tau, \Delta X_{n}\right\rangle} \mid \mathscr{F}_{n-1}\right), \quad \tau \in \mathbf{R}^{d} .
\end{aligned}
$$

(Эти неравенства проверяем для $n=N, \ldots, 1$, спускаясь от $N$ к 1.)

Теорема 5.2. Рассмотрим безарбитражную модель вида (5.1).

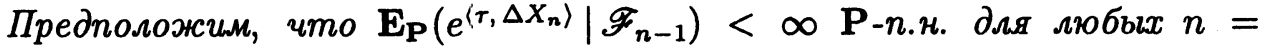
$1, \ldots, N, \tau \in \mathbf{R}^{d}$. Тогда минимум $H(\mathbf{Q}, \mathbf{P})$ по множеству $\mathscr{M}^{a}$ достигается на единственной мере

$$
\mathbf{Q}_{*}=\text { const } \cdot \exp \left\{\sum_{n=1}^{N}\left\langle\tau_{n}^{*}, \Delta X_{n}\right\rangle\right\} \mathbf{P}
$$


где случайнье величины $\tau_{n}^{*}$ определяются равенством (5.2). При этом

$$
H\left(\mathbf{Q}_{*}, \mathbf{P}\right)=-\ln \mathbf{E}_{\mathbf{P}} \exp \left\{\sum_{n=1}^{N}\left\langle\tau_{n}^{*}, \Delta X_{n}\right\rangle\right\}
$$

Д ок а з а тель ство. Предположим, что существует мера $\mathbf{Q} \in \mathscr{M}^{a}$ такая, что $H(\mathbf{Q}, \mathbf{P})<H\left(\mathbf{Q}_{*}, \mathbf{P}\right)$. Положим

$$
\begin{aligned}
U_{n} & =\frac{d \mathbf{Q} \mid \mathscr{F}_{n}}{d \mathbf{P} \mid \mathscr{F}_{n}}, \quad n=0, \ldots, N, \\
V_{n} & =\frac{e^{\left\langle\tau_{n}^{*}, \Delta X_{n}\right\rangle} \mathbf{E}_{\mathbf{P}}\left(\exp \left\{\sum_{i=n+1}^{N}\left\langle\tau_{i}^{*}, \Delta X_{i}\right\rangle\right\} \mid \mathscr{F}_{n}\right)}{\mathbf{E}_{\mathbf{P}}\left(\exp \left\{\sum_{i=n}^{N}\left\langle\tau_{i}^{*}, \Delta X_{i}\right\rangle\right\} \mid \mathscr{F}_{n-1}\right)}, \quad n=0, \ldots, N,
\end{aligned}
$$

где $\mathscr{F}_{-1}:=\{\varnothing, \Omega\}, \Delta X_{0}:=0$. Рассмотрим меры

$$
\mathbf{Q}_{n}=U_{n} V_{n+1} \cdots V_{N} \mathbf{P}, \quad n=-1, \ldots, N,
$$

где $U_{-1}:=1$. Докажем, что для любого $k=0, \ldots, N$

$$
H\left(\mathbf{Q}_{k-1}, \mathbf{P}\right) \leqslant H\left(\mathbf{Q}_{k}, \mathbf{P}\right)
$$

и

$$
\mathbf{E}_{\mathbf{P}}\left(V_{k} \cdots V_{N} \ln V_{k} \cdots V_{N} \mid \mathscr{F}_{k-1}\right)=Y_{k-1},
$$

где

$$
Y_{n}=-\ln \mathbf{E}_{\mathbf{P}}\left(\exp \left\{\sum_{i=n+1}^{N}\left\langle\tau_{i}^{*}, \Delta X_{i}\right\rangle\right\} \mid \mathscr{F}_{n}\right), \quad n=-1, \ldots, N .
$$

Предположим, что эти утверждения уже доказаны для $k=n+$ $1, \ldots, N$. Докажем их для $k=n$. Имеем

$$
\begin{aligned}
H\left(\mathbf{Q}_{n-1}, \mathbf{P}\right)= & \mathbf{E}_{\mathbf{P}} U_{n-1} V_{n} \cdots V_{N} \ln U_{n-1} V_{n} \cdots V_{N} \\
= & \mathbf{E}_{\mathbf{P}} U_{n-1} V_{n} \cdots V_{N} \ln V_{n+1} \cdots V_{N}+\mathbf{E}_{\mathbf{P}} U_{n-1} V_{n} \cdots V_{N} \ln V_{n} \\
& +\mathbf{E}_{\mathbf{P}} U_{n-1} V_{n} \cdots V_{N} \ln U_{n-1} \\
= & \mathbf{E}_{\mathbf{P}} \mathbf{E}_{\mathbf{P}}\left(U_{n-1} V_{n} \cdots V_{N} \ln V_{n+1} \cdots V_{N} \mid \mathscr{F}_{n}\right) \\
& +\mathbf{E}_{\mathbf{P}} \mathbf{E}_{\mathbf{P}}\left(U_{n-1} V_{n} \cdots V_{N} \ln V_{n} \mid \mathscr{F}_{n}\right) \\
& +\mathbf{E}_{\mathbf{P}} \mathbf{E}_{\mathbf{P}}\left(U_{n-1} V_{n} \cdots V_{N} \ln U_{n-1} \mid \mathscr{F}_{n-1}\right) \\
= & \mathbf{E}_{\mathbf{P}} U_{n-1} V_{n} Y_{n}+\mathbf{E}_{\mathbf{P}} U_{n-1} V_{n} \ln V_{n}+\mathbf{E}_{\mathbf{P}} U_{n-1} \ln U_{n-1} .
\end{aligned}
$$

Здесь мы воспользовались равенством (5.7) для $k=n+1$, а также очевидным свойством $\mathbf{E}_{\mathbf{P}}\left(V_{i} \mid \mathscr{F}_{j}\right)=1$ при $i>j$. Аналогично

$$
H\left(\mathbf{Q}_{n}, \mathbf{P}\right)=\mathbf{E}_{\mathbf{P}} U_{n-1} W_{n} Y_{n}+\mathbf{E}_{\mathbf{P}} U_{n-1} W_{n} \ln W_{n}+\mathbf{E}_{\mathbf{P}} U_{n-1} \ln U_{n-1},
$$

где $W_{n}=I\left(U_{n-1}>0\right) U_{n} / U_{n-1}$. Пусть $\mathbf{P}_{\omega}$ обозначает условное распределение $\operatorname{Law}\left(\Delta X_{n}, Y_{n}, \tau_{n}^{*}, U_{n}, V_{n}, W_{n} \mid \mathscr{F}_{n-1}\right)(\omega)$. Обозначим через $\widetilde{\Delta X}_{n}$, 
$\tilde{Y}_{n}, \widetilde{\tau}_{n}^{*}, \widetilde{U}_{n}, \widetilde{V}_{n}, \widetilde{W}_{n}$ координатные отображения на $\mathbf{R}^{2 d+4}\left(\widetilde{\Delta X}_{n}\right.$ и $\widetilde{\tau}_{n}^{*}$ принимают значения в $\mathbf{R}^{d}$, в то время как остальные четыре отображения принимают значения в $\mathbf{R}$ ). Из равенства (5.7) с $k=n+1$ следует, что $Y_{n} \geqslant 0$. Используя равенство

$$
\begin{aligned}
& \mathbf{E}_{\mathbf{P}}\left(e^{\left\langle\tau, \Delta X_{n}\right\rangle-Y_{n}} \mid \mathscr{F}_{n-1}\right) \\
& =\mathbf{E}_{\mathbf{P}}\left(e^{\left\langle\tau, \Delta X_{n}\right\rangle} \mathbf{E}_{\mathbf{P}}\left(\exp \left\{\sum_{i=n+1}^{N}\left\langle\tau_{i}^{*}, \Delta X_{i}\right\rangle\right\} \mid \mathscr{F}_{n}\right) \mid \mathscr{F}_{n-1}\right) \\
& =\mathbf{E}_{\mathbf{P}}\left(\exp \left\{\left\langle\tau, \Delta X_{n}\right\rangle+\sum_{i=n+1}^{N}\left\langle\tau_{i}^{*}, \Delta X_{i}\right\rangle\right\} \mid \mathscr{F}_{n-1}\right), \quad \tau \in \mathbf{R}^{d},
\end{aligned}
$$

получаем

$$
\widetilde{\tau}_{n}^{*}=\underset{\tau \in \mathbf{R}^{d}}{\arg \min } \mathbf{E}_{\mathbf{P}_{\omega}} e^{\left\langle\tau, \widetilde{\left.\Delta X_{n}\right\rangle-\widetilde{Y}_{n}}\right.} \quad \text { для } \quad \mathbf{P} \text {-п.в. } \omega .
$$

Равенство (5.5) можно переписать в виде

$$
\tilde{V}_{n}=\frac{e^{\left(\widetilde{\tau}_{n}^{*}, \widetilde{\Delta X}_{n}\right\rangle-\widetilde{Y}_{n}}}{\mathbf{E}_{\mathbf{P}_{\omega}} e^{\left(\widetilde{\tau}_{n}^{*}, \widetilde{\Delta X}_{n}\right)-\widetilde{Y}_{n}}} \quad \text { для } \quad \text { Р-П.В. } \omega .
$$

Из условия $\mathbf{E}_{\mathbf{P}_{\omega}}\left(e^{\left\langle\tau, \Delta X_{n}\right\rangle-Y_{n}} \mid \mathscr{F}_{n-1}\right)<\infty \mathbf{P}$-п.н. вытекает, что

$$
\mathbf{E}_{\mathbf{P}_{\omega}}\left\|\widetilde{\Delta X}_{n}\right\| e^{\left(\widetilde{\tau}_{n}^{*}, \widetilde{\Delta X}_{n}\right\rangle-\widetilde{Y}_{n}}<\infty \quad \text { для } \quad \mathbf{P} \text {-п.в. } \omega .
$$

Из неравенства $Y_{n} \geqslant 0$ следует, что

$$
\mathbf{E}_{\mathbf{P}_{\omega}} \widetilde{Y}_{n} e^{\left(\widetilde{\tau}_{n}^{*}, \widetilde{\Delta X}_{n}\right)-\widetilde{Y}_{n}}<\infty \quad \text { для } \quad \mathbf{P} \text {-п.в. } \omega .
$$

Согласно определению $W_{n}$,

$$
\mathbf{E}_{\mathbf{P}_{\omega}} \widetilde{W}_{n}=1 \text { для } \mathbf{P} \text {-п.в. } \omega \text { из множества }\left\{U_{n-1}>0\right\} .
$$

Условие $\mathbf{Q} \in \mathscr{M}^{a}$ влечет неравенство

$$
\mathbf{E}_{\mathbf{Q}}\left(\left\|\Delta X_{n}\right\| \mid \mathscr{F}_{n-1}\right)=\mathbf{E}_{\mathbf{P}}\left(W_{n}\left\|\Delta X_{n}\right\| \mid \mathscr{F}_{n-1}\right)<\infty \quad \text { P-п.н. }
$$

(см. $[16$, гл. II, §1c]). Отсюда получаем

$$
\mathbf{E}_{\mathbf{P}_{\omega}}\left\|\widetilde{\Delta X}_{n}\right\| \widetilde{W}_{n}<\infty \quad \text { для } \mathbf{P} \text {-п.в. } \omega \text { из множества }\left\{U_{n-1}>0\right\} \text {. }
$$

Используя свойства (5.11)-(5.15) и лемму 2.1, можем написать

$$
\begin{aligned}
& -\mathbf{E}_{\mathbf{P}_{\omega}} \widetilde{V}_{n}\left\langle\widetilde{\tau}_{n}^{*}, \widetilde{\Delta X}_{n}\right\rangle+\mathbf{E}_{\mathbf{P}_{\omega}} \tilde{V}_{n} \tilde{Y}_{n}+\mathbf{E}_{\mathbf{P}_{\omega}} \widetilde{V}_{n} \ln \widetilde{V}_{n} \\
& \quad \leqslant-\mathbf{E}_{\mathbf{P}_{\omega}} \widetilde{W}_{n}\left\langle\widetilde{\tau}_{n}^{*}, \widetilde{\Delta X}_{n}\right\rangle+\mathbf{E}_{\mathbf{P}_{\omega}} \widetilde{W}_{n} \tilde{Y}_{n}+\mathbf{E}_{\mathbf{P}_{\omega}} \widetilde{W}_{n} \ln \widetilde{W}_{n}
\end{aligned}
$$

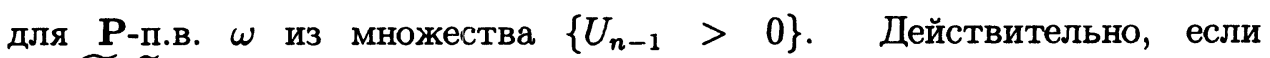
$\mathbf{E}_{\mathbf{P}_{\omega}} \widetilde{W}_{n} \widetilde{Y}_{n}=\infty$, то это неравенство очевидно; иначе оно следует из леммы 2.1. С учетом (5.10) и (5.11), получаем

$$
\mathbf{E}_{\mathbf{P}_{\omega}} \tilde{V}_{n} \widetilde{\Delta X}_{n}=0 \quad \text { для } \quad \text { Р-п.в. } \omega .
$$


Согласно неравенству

$$
\mathbf{E}_{\mathbf{P}}\left(W_{n} \Delta X_{n} \mid \mathscr{F}_{n-1}\right)=I\left(U_{n-1}>0\right) \mathbf{E}_{\mathbf{Q}}\left(\Delta X_{n} \mid \mathscr{F}_{n-1}\right)=0,
$$

имеем

$\mathbf{E}_{\mathbf{P}_{\omega}} \widetilde{\Delta X}_{n} \widetilde{W}_{n}=0 \quad$ для $\quad$ P-п.в. $\omega$ из множества $\left\{U_{n-1}>0\right\} .(5.18)$ Сравнивая равенства (5.16)-(5.18), получаем

$$
\mathbf{E}_{\mathbf{P}_{\omega}} \tilde{V}_{n} \tilde{Y}_{n}+\mathbf{E}_{\mathbf{P}_{\omega}} \tilde{V}_{n} \ln \tilde{V}_{n} \leqslant \mathbf{E}_{\mathbf{P}_{\omega}} \widetilde{W}_{n} \tilde{Y}_{n}+\mathbf{E}_{\mathbf{P}_{\omega}} \widetilde{W}_{n} \ln \widetilde{W}_{n}
$$

для Р-п.в. $\omega$ из множества $\left\{U_{n-1}>0\right\}$. Учитывая $(5.8)$ и $(5.9)$, приходим к неравенству $H\left(\mathbf{Q}_{n-1}, \mathbf{P}\right) \leqslant H\left(\mathbf{Q}_{n}, \mathbf{P}\right)$. Далее,

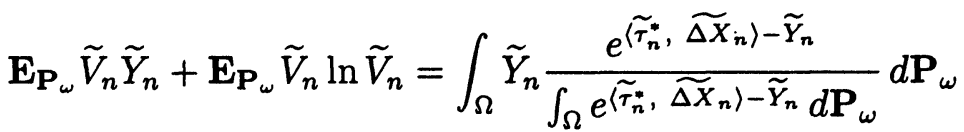

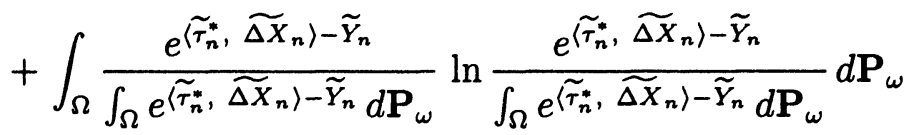

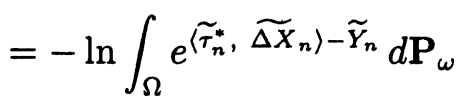

для Р-п.в. $\omega$ (мы воспользовались равенством (5.17)). Отсюда

$$
\begin{aligned}
\mathbf{E}_{\mathbf{P}} & \left(V_{n} \cdots V_{N} \ln V_{n} \cdots V_{N} \mid \mathscr{F}_{n-1}\right) \\
= & \mathbf{E}_{\mathbf{P}}\left(V_{n} \cdots V_{N} \ln V_{n+1} \cdots V_{N} \mid \mathscr{F}_{n-1}\right)+\mathbf{E}_{\mathbf{P}}\left(V_{n} \cdots V_{N} \ln V_{n} \mid \mathscr{F}_{n-1}\right) \\
= & \mathbf{E}_{\mathbf{P}}\left(V_{n} Y_{n} \mid \mathscr{F}_{n-1}\right)+\mathbf{E}_{\mathbf{P}}\left(V_{n} \ln V_{n} \mid \mathscr{F}_{n-1}\right) \\
= & -\ln \mathbf{E}_{\mathbf{P}}\left(e^{\left\langle\tau_{n}^{*}, \Delta X_{n}\right\rangle-Y_{n}} \mid \mathscr{F}_{n-1}\right)=Y_{n-1} .
\end{aligned}
$$

Итак, мы доказали (5.6) и (5.7) для $k=0, \ldots, N$.

Заметим, что $\mathbf{Q}_{*}=\mathbf{Q}_{-1}$. C помощью (5.17) получаем

$$
\begin{aligned}
\mathbf{E}_{\mathbf{Q}}\left(\Delta X_{n} \mid \mathscr{F}_{n-1}\right)=\frac{\mathbf{E}_{\mathbf{P}}\left(V_{0} \cdots V_{N} \Delta X_{n} \mid \mathscr{F}_{n-1}\right)}{\mathbf{E}_{\mathbf{P}}\left(V_{0} \cdots V_{N} \mid \mathscr{F}_{n-1}\right)} \\
=\frac{\mathbf{E}_{\mathbf{P}}\left(\mathbf{E}_{\mathbf{P}}\left(V_{n} \cdots V_{N} \Delta X_{n} \mid \mathscr{F}_{n}\right) \mid \mathscr{F}_{n-1}\right)}{\mathbf{E}_{\mathbf{P}}\left(V_{n} \cdots V_{N} \mid \mathscr{F}_{n-1}\right)}=\frac{\mathbf{E}_{\mathbf{P}}\left(V_{n} \Delta X_{n} \mid \mathscr{F}_{n-1}\right)}{\mathbf{E}_{\mathbf{P}}\left(V_{n} \cdots V_{N} \mid \mathscr{F}_{n-1}\right)}=0 .
\end{aligned}
$$

Следовательно, $\mathbf{Q}_{*} \in \mathscr{M}^{a}$. Поскольку $\mathbf{Q}=\mathbf{Q}_{N}$, заключаем, что $H\left(\mathbf{Q}_{*}, \mathbf{P}\right) \leqslant H(\mathbf{Q}, \mathbf{P})$ и

$H\left(\mathbf{Q}_{*}, \mathbf{P}\right)=\mathbf{E}_{\mathbf{P}}\left(V_{0} \cdots V_{N} \ln V_{0} \cdots V_{N}\right)=Y_{-1}=-\ln \mathbf{E}_{\mathbf{P}} \exp \left\{\sum_{n=1}^{N}\left\langle\tau_{n}^{*}, \Delta X_{n}\right\rangle\right\}$.

Итак, минимум $H(\mathbf{Q}, \mathbf{P})$ по множеству $\mathscr{M}^{a}$ достигается на единственной мере $\mathbf{Q}_{*}$. Единственность минимизирующей меры следует из выпуклости множества $\mathscr{M}^{a}$ и строгой выпуклости функции $x \mapsto x \ln x$. 
З а м е ч а н и е. Описанный выше метод построения ближайшей по энтропии мартингальной меры схож с методом построения некоторой мартингальной меры при помощи условного преобразования Эшера; cм. [14].

Следствие 5.1. Рассмотрим безарбитражную модель вида (5.1). Предположим, ито $\Delta X_{n}$ не зависит от $\mathscr{F}_{n-1}$ для любого $n=1, \ldots, N$ $u \mathbf{E}_{\mathbf{P}} e^{\left(\tau, \Delta X_{n}\right)}<\infty$ для любых $n=1, \ldots, N, \tau \in \mathbf{R}^{d}$. Тогда при каждом $n=1, \ldots, N$ найдется точка $\tau_{n}^{*} \in \mathbf{R}^{d}$, в которой достигается минимум функиии $\varphi_{n}(\tau)=\mathbf{E}_{\mathbf{P}} e^{\left\langle\tau, \Delta X_{n}\right\rangle}$. Минимум $H(\mathbf{Q}, \mathbf{P})$ по множеству $\mathscr{M}^{a}$ достигается на единственной мере $\mathbf{Q}_{*}=$ const. $\exp \left\{\sum_{n=1}^{N}\left\langle\tau_{n}^{*}, \Delta X_{n}\right\rangle\right\} \mathbf{P}$.

Д о к а з а т е л с с в о. Достаточно заметить, что условные математические ожидания в (5.2) совпадают с безусловными. . Теперь утверждение вытекает из теоремы 5.2.

Следствие 5.2. Рассмотрим одномерную безарбитражную модель вида (5.1). Предположим, что $X_{n}=e^{Y_{n}}$, где $\Delta Y_{n}=Y_{n}-Y_{n-1}$ не зависит от $\mathscr{F}_{n-1}$ для любого $n=1, \ldots, N u \mathbf{E}_{\mathbf{P}} e^{\tau\left(e^{\Delta Y_{n}}-1\right)}<\infty$ для любих $n=1, \ldots, N, \tau \in \mathbf{R}$. Тогда при каждом $n=1, \ldots, N$ найдется точка $\lambda_{n}^{*} \in \mathbf{R}$, в которой достигается минимум функиии $\varphi_{n}(\lambda)=\mathbf{E}_{\mathbf{P}} e^{\lambda\left(e^{\Delta Y_{n}}-1\right)}$. Минимум $H(\mathbf{Q}, \mathbf{P})$ по множеству $\mathscr{M}^{a}$ достигается на единственной мере

$$
\mathbf{Q}_{*}=\text { const } \cdot \exp \left\{\sum_{n=1}^{N} \frac{\lambda_{n}^{*}}{X_{n-1}} \Delta X_{n}\right\} \mathbf{P} .
$$

Д о к а з а т е л ь с т в о. Докажем, что для любого $k=N, \ldots, 1$ случайная величина $\tau_{k}^{*}$, определенная в (5.2), совпадает с $\lambda_{k}^{*} / X_{k-1}$. Предположим, что это утверждение уже доказано для $k=n+1, \ldots, N$. Докажем его для $k=n$. Имеем

$$
\begin{aligned}
& \mathbf{E}_{\mathbf{P}}\left(\exp \left\{\tau \Delta X_{n}+\sum_{i=n+1}^{N} \tau_{i}^{*} \Delta X_{i}\right\} \mid \mathscr{F}_{n-1}\right) \\
& =\mathbf{E}_{\mathbf{P}}\left(e^{\tau \Delta X_{n}} \mathbf{E}_{\mathbf{P}}\left(\exp \left\{\sum_{i=n+1}^{N} \lambda_{i}^{*}\left(e^{\Delta Y_{i}}-1\right)\right\} \mid \mathscr{F}_{n}\right) \mid \mathscr{F}_{n-1}\right) \\
& =\mathbf{E}_{\mathbf{P}} \exp \left\{\sum_{i=n+1}^{N} \lambda_{i}^{*}\left(e^{\Delta Y_{i}}-1\right)\right\} \mathbf{E}_{\mathbf{P}}\left(e^{\tau \Delta X_{n}} \mid \mathscr{F}_{n-1}\right) \\
& =\mathbf{E}_{\mathbf{P}} \exp \left\{\sum_{i=n+1}^{N} \lambda_{i}^{*}\left(e^{\Delta Y_{i}}-1\right)\right\} \varphi_{n}\left(\tau X_{n-1}\right) .
\end{aligned}
$$

Итак, мы проверили, что $\tau_{k}^{*}=\lambda_{k}^{*} / X_{k-1}$ для $k=N, \ldots, 1$. Теперь требуемое утверждение вытекает из теоремы 5.2. 
5.3. Максимизация экспоненциальной полезности. Как уже отмечалось, проблема нахождения ближайшей по энтропии мартингальной меры двойственна проблеме максимизации экспоненциальной полезности.

О п р е д е л е и е 5.3. Экспоненциальной полезностью стратегии $\pi$ называется число $U(\pi)=-\mathbf{E}_{\mathbf{P}} e^{-X_{N}^{\pi}}$.

Теорема 5.3. Рассмотрим безарбитражную модель вида (5.1). Предположим, что $\mathbf{E}_{\mathbf{P}}\left(e^{\left\langle\tau, \Delta X_{n}\right\rangle} \mid \mathscr{F}_{n-1}\right)<\infty$ P-п.н. для любьх $n=1, \ldots, N, \tau \in \mathbf{R}^{d}$. Пусть $x \in \mathbf{R}$. Тогда максимум $U(\pi)$ по всем стратегиям $\pi$ с начальным капиталом $x$ достигается на единственной стратегии $\pi_{*}=\left(x, H^{*}\right)$, где $H_{n}^{*}=-\tau_{n}^{*}$, a случайные величины $\tau_{n}^{*}$ определяются равенством (5.2).

Д о к а з а т е л с т в о. Предположим, что существует стратегия $\pi=(x, H)$ такая, что $U(\pi)>U\left(\pi^{*}\right)$. Тогда

$$
\mathbf{E}_{\mathbf{P}} \exp \left\{-\sum_{i=1}^{N}\left\langle H_{i}, \Delta X_{i}\right\rangle\right\}<\mathbf{E}_{\mathbf{P}} \exp \left\{-\sum_{i=1}^{N}\left\langle H_{i}^{*}, \Delta X_{i}\right\rangle\right\} .
$$

Рассмотрим стратегии $\pi^{(n)}=\left(x, H^{(n)}\right), n=0, \ldots, N$, определенные по формуле

$$
H_{i}^{(n)}=\left\{\begin{array}{lll}
H_{i}, & \text { если } & i \leqslant n, \\
H_{i}^{*}, & \text { если } i>n .
\end{array}\right.
$$

Тогда для любого $n=1, \ldots, N$ выполнено

$$
\begin{aligned}
& \mathbf{E}_{\mathbf{P}}\left(\exp \left\{-\sum_{i=1}^{N}\left\langle H_{i}^{(n-1)}, \Delta X_{i}\right\rangle\right\} \mid \mathscr{F}_{n-1}\right)=\exp \left\{-\sum_{i=1}^{n-1}\left\langle H_{i}, \Delta X_{i}\right\rangle\right\} \\
& \quad \times \mathbf{E}_{\mathbf{P}}\left(\exp \left\{-\left\langle H_{n}^{*}, \Delta X_{n}\right\rangle-\sum_{i=n+1}^{N}\left\langle H_{i}^{*}, \Delta X_{i}\right\rangle\right\} \mid \mathscr{F}_{n-1}\right) \\
& \leqslant \exp \left\{-\sum_{i=1}^{n-1}\left\langle H_{i}, \Delta X_{i}\right\rangle\right\} \\
& \times \mathbf{E}_{\mathbf{P}}\left(\exp \left\{-\left\langle H_{n}, \Delta X_{n}\right\rangle-\sum_{i=n+1}^{N}\left\langle H_{i}^{*}, \Delta X_{i}\right\rangle\right\} \mid \mathscr{F}_{n-1}\right) \\
&= \mathbf{E}_{\mathbf{P}}\left(\exp \left\{-\sum_{i=1}^{N}\left\langle H_{i}^{(n)}, \Delta X_{i}\right\rangle\right\} \mid \mathscr{F}_{n-1}\right),
\end{aligned}
$$

откуда получаем неравенство $U\left(\pi^{(n-1)}\right) \leqslant U\left(\pi^{(n)}\right)$. Поскольку $\pi_{*}=\pi^{(0)}$ и $\pi=\pi^{(N)}$, приходим к противоречию. Итак, минимум $U(\pi)$ достигается на стратегии $\pi_{*}$.

Единственность стратегии, максимизирующей $U(\pi)$, вытекает из строгой выпуклости функции $x \mapsto-e^{-x}$. 
З а м е ч а н и е. Справедливо равенство

$$
-H\left(\mathbf{Q}_{*}, \mathbf{P}\right)=x+\ln \left(-U\left(\pi_{*}\right)\right),
$$

где $\mathbf{Q}_{*}$ - мера, определенная в (5.3).

\section{СПИСОК ЛИТЕРАТУРЫ}

1. Bucklew J. Large Deviation Techniques in Decision, Simulation, and Estimation. New York: Wiley, $1990,270 \mathrm{p}$.

2. Cover T.M., Thomas J. A. Elements of Information Theory. New York: Wiley, 1991, $542 \mathrm{p}$.

3. Csiszar I. I-divergence geometry of probability distributions and minimization problems. - Ann. Probab., 1975, v. 3, № 1, p. 146-158.

4. Delbaen F., Grandits P., Rheinländer T., Samperi D., Schweizer M., Stricker C. Exponential hedging and entropic penalties. - Math. Finance, 2002, v. 12, №2, p. 99-123.

5. Dellacherie C., Meyer P.-A. Probabilités et potentiel. V. I. Paris: Hermann, 1976.

6. Föllmer H., Schied A. Stochastic Finance. An Introduction in Discrete Time. Berlin: de Gruyter, 2002, 422 p.

7. Frittelli $M$. The minimal entropy martingale measure and the valuation problem in incomplete markets. $\rightarrow$ Math. Finance, 2000 , v. 10, № 1, p. 39-52.

8. Fujiwara $T$., Miyahara' $Y$. The minimal entropy martingale measures for geometric Lévy processes. - Finance Stoch., 2003, v. 7, № 4, p. 509-531.

9. Goll T., Rüschendorf L. Minimax and minimal distance martingale measures and their relationship to portfolio optimization. - Finance Stoch., 2001, v. 5, № 4, p. 557-581.

10. Jaynes E. T. Papers on Probability, Statistics and Statistical Physics. Dordrecht: Reidel, 1983, $434 \mathrm{p}$.

11. Лаґдау Л. Д., Лифиич Е. М. Теоретическая физика. Т. 5: Статистическая физика. М.: Наука, 1995.

12. Maslov V.P. The notions of enropy, Hamiltonian, temperature, and thermodynamical limit in probability theory used for solving model problems in econophysics. Russian J. Math. Phys., 2002, v. 9, № 4, p. 437-445.

13. Miyahara Y., Novikov A. Geometric Lévy process pricing model. - Труды МИАН, 2002 , т. 237 , c. $185-200$.

14. Rogers L.C. G. Equivalent martingale measures and no-arbitrage. - Stochastics Stochastics Rep., 1995, v. 51, № 1-2, p. 41-49.

15. Schachermayer $W$. Optimal investment in incomplete markets when wealth may become negative. - Ann. Appl. Probab., 2001, v. 11, №3, p. 694-734.

16. Ширяев A. Н. Основы стохастической финансовой математики. Т. 1, 2. М.: Фазис, 1998,544 c., 512 c.

17. Стратонович Р. Л. Теория информации. М.: Советское радио, 1975.

Поступила в редакцию 26.III. 2003 